\title{
An extremely indolent T-cell leukemia: an 18-year follow-up
}

\author{
Samuel Adediran, MD, ${ }^{a}$ Dennis Cornfield, MD, ${ }^{\mathrm{b}}$ Adam Bagg, MD, ${ }^{\mathrm{c}}$ and Nicole Agostino, \\ $\mathrm{DO}^{\mathrm{a}, \mathrm{d}}$
}

Lehigh Valley Hospital, Departments of a Medicine, ${ }^{b}$ Pathology and Laboratory Medicine, and division of Hematology/Medical Oncology, Allentown, Pennsylvania, and 'University of Pennsylvania Medical Center, Department of Pathology and Laboratory Medicine, Philadelphia

$\mathrm{T}$ -cell prolymphocytic leukemia (T-PLL) is a rare malignancy that comprises about $2 \%$ of all mature lymphoid neoplasms. Patients usually present with prominent peripheral blood lymphocytosis, splenomegaly, hepatomegaly, lymphadenopathy, B symptoms, and occasionally with skin lesions. ${ }^{1}$ The disease follows an aggressive clinical course with rapid progression and typically has a median survival of less than 1 year. In some cases, the disease is indolent for a period of time before becoming aggressive. ${ }^{2}$ In 2002, 7 years after initial diagnosis in 1995, the case discussed herein was reported as a rare, indolent form of T-PLL.3 We now present 11 additional years of follow-up of this case, during which time the patient remained asymptomatic with respect to his lymphoid neoplasm.

\section{Case presentation and summary}

The patient was a 73-year-old man who had an unusually indolent form of T-PLL. ${ }^{3}$ His initial evaluation at the time of diagnosis in 1995 had shown peripheral blood lymphocytosis with small, matureappearing lymphocytes. There was no evidence of hepatosplenomegaly, lymphadenopathy, or skin lesions. A flow cytometric analysis of the peripheral blood revealed lymphocytes with a CD4(+)CD8(-) postthymic helper T-cell immunophenotype (see Table for a summary of the findings reported in 2002). For the next 11 years (18 years after the initial diagnosis in 1995), the patient remained asymptomatic with regard to his hematologic disorder. In 2011, he underwent transcatheter aortic valve replacement for severe aortic stenosis. The patient was diagnosed with cholestatic hepatitis with grade 1 hepatic fibrosis in 2011.

In 2013, the patient was found to have a white blood cell count of $112.4 \times 10^{9} / \mathrm{L}$, with $90 \%$ lymphocytes (normal, 4.5-10.5 × 10\% $/ \mathrm{L}, 34 \% \mathrm{lym}^{-}$ phocyte). He reported mild fatigue but no fever, weight loss, or night sweats. A physical examination revealed a mildly tense protuberant abdomen, palpable bilateral cervical lymph nodes, and palpable right axillary lymph nodes. There was $2+$ pitting edema of the lower extremities (range, $1+$ to $4+$ $[1+=\leq 2 \mathrm{~mm} ; 4+=6-8 \mathrm{~mm}])$. His hemoglobin was $13.2 \mathrm{~g} / \mathrm{dL}$ (normal, 12.6-17.4 g/dL), and platelet count was $135 \times 10^{9} / \mathrm{L}$ (normal, 150-400 × 10\% $/ \mathrm{L}$ ). Liver function tests showed the following levels: alanine transaminase (ALT), $124 \mathrm{U} / \mathrm{L}$ (normal, $<40$ $\mathrm{U} / \mathrm{L}$ ); aspartate aminotransferase (AST), $72 \mathrm{U} / \mathrm{L}$ (normal, <40 U/L); and alkaline phosphatase, 180 U/L (normal, <136 U/L).

A computed-tomography (CT) scan showed extensive lymphadenopathy, slight splenomegaly, a moderate-sized right pleural effusion, and a large amount of abdominal and pelvic ascites. An examination of the peripheral smear showed a marked lymphocytosis that consisted of small-to-mildly enlarged lymphocytes with condensed nuclear chromatin, and occasional prolymphocytes were present (Figure 1). A bone marrow biopsy revealed moderately hypercellular marrow with numerous patches of small, mature-appearing lymphocytes in a background of normal hematopoiesis. Flow cytometry of the bone marrow aspirate showed predominantly T-cell lymphocytes with a CD3(+)CD4(+)CD5(+) CD8(-) immunophenotype. Cytogenetic analysis revealed a 45,X,-Y[18], XY[2] karyotype (the failure to reproduce the complex karyotype noted 11 years earlier may have been related to technical issues). Ascitic fluid white cell count was 130/cmm ( $<500 /$ $\mathrm{cmm}$ ), with a majority lymphocytes. Cytospin

Accepted for publication January 13, 2015. Disclosures: The authors have no disclosures. Correspondence Samuel Adediran, MD; Samuel.adediran@lvhn.org. JCSO 2016;14:76-78. @2016 Frontline Medical Communications. doi: 10.12788/icso.0190. 


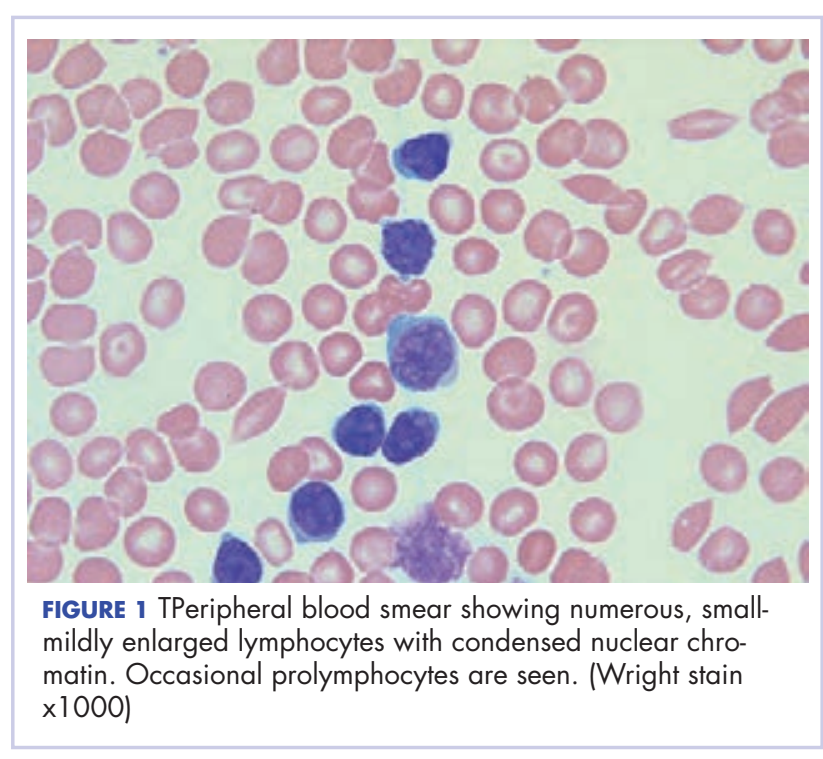

showed numerous clusters of mostly enlarged lymphocytes with moderately dispersed nuclear chromatin and frequently a prominent nucleolus, morphologically consistent with prolymphocytes (Figure 2). Flow cytometric findings were similar to those of the bone marrow biopsy.

Based on our examination of the patient and the results of his tests and assessments, we believed the patient's ascites and pleural effusion were caused predominantly by his progressive T-cell lymphoproliferatve disorder. He was treated with 1 cycle of alemtuzumab. He experienced normalization of his white blood cell count and differential (WBC, $10.9 \times 10^{9} / \mathrm{L}$, with $34 \%$ lymphocytes) and regression of his lymphadenopathy. However, he continued to have recurrent ascites requiring frequent therapeutic para-

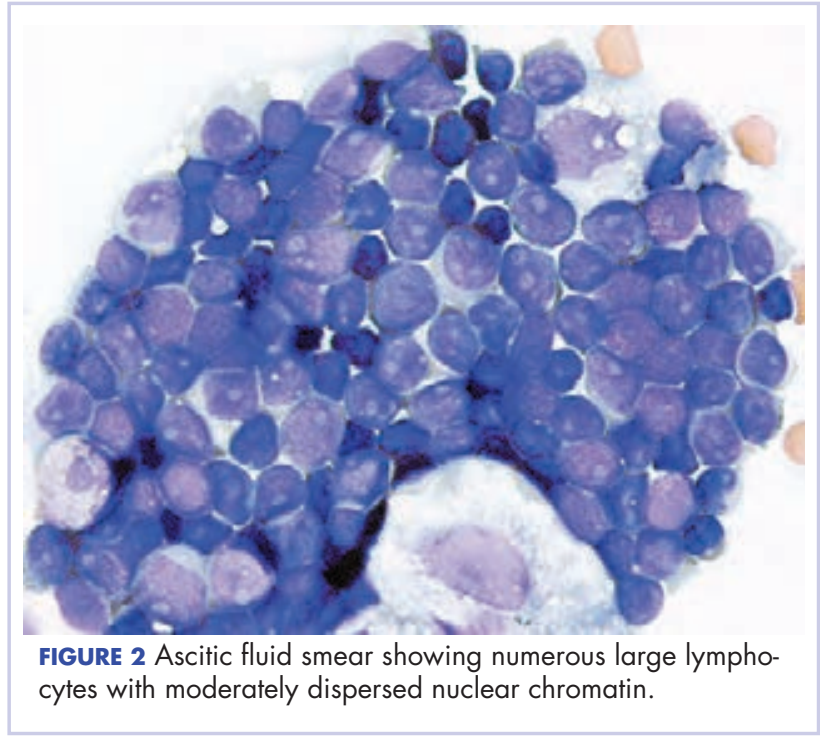

centesis. His global health declined steadily over the ensuing several weeks, and he eventually died, 18 years after his disease was first recognized.

\section{Discussion}

This report represents 11 additional years of follow-up to the original case report of 2002, at which time this patient had already gone 7 years from the time of his diagnosis of T-PLL without the need for any treatment. ${ }^{3} \mathrm{~A}$ total of 18 years of indolent disease is virtually unheard of in a T-cell lymphoproliferative disorder (T-LPD). It emphasizes the uniqueness of this case and raises questions, already posed in the 2002 report, regarding proper classification.

The Revised European American Lymphoma (REAL)

TABLE 1 Summary of findings in original 2002 report of the current case

\section{Tests}

Peripheral blood WBC count

\section{Results}

$12.7 \times 109 / \mathrm{L}$, with $73 \%$ lymphocytes (normal, 4.5-10.5× 109/L, $34 \%$ lymphocyte)

Peripheral blood Wright-stained smear

Lymphocytes with regular and irregular nuclear membranes, rare nonprominent nucleoli and occasional cytoplasmic blebs

Lymphocyte morphology by electron microscopy

Peripheral blood: flow cytometric immunophenotype of T-cell clone

Cytogenetics study of peripheral blood$$
\text { Cy }
$$

\footnotetext{
PCR for T-cell receptor gamma gene rearrangement
} $\mathrm{PCR}$, polymerase chain reaction; WBC, white blood cell
Lymphoctes frequently exhibit nucleoli

$\mathrm{CD} 2(+), \mathrm{CD} 3(+), \mathrm{CD} 4(+), \mathrm{CD} 5(+), \mathrm{CD} 7(+), \mathrm{CD} 45 \mathrm{RA}(+)$, CD45RO(+), CD62L(+), TCR alpha/beta(+)

Abnormal subclones with complex abnormalities [7 cells: 47,XY, +8, inv(1)(p12q26), del(1 1)(p13p15); 6 cells: $X Y$,inv(10)(p12q26), del(11)(p13p15); 4 cells: $47, X Y, \operatorname{inv}(10)$ (p12q26), del(11)(p13p15)+marker; 3 cells: 47,Y,-X, $+8, \operatorname{inv}(1)$ (p12q26), del(1 1)(p12p15)+marker].

Monoclonal rearrangement present 
classification of lymphomas of 1994 included T-cell chronic lymphocytic leukemia (CLL)/PLL as a bona fide entity, with most cases exhibiting morphologic features of typical prolymphocytes (T-PLL) and fewer cases showing small lymphocytes with condensed nuclear chromatin and inconspicuous or absent nucleoli by light microscopy (T-CLL). ${ }^{4}$ However, T-CLL disappeared in the first (2001) and most recent (2008) World Health Organization (WHO) classifications of lymphoma. T-CLL was subsumed into the T-PLL category because of the fairly uniform clinical presentation and biologic behavior of most cases of T-CLL/T-PLL, namely prominent lymphocytosis and splenomegaly at diagnosis, poor response to chemotherapy, and a median survival of less than 1 year. ${ }^{5,6}$ In a 1991 British series of 78 patients with T-PLL, 15 patients (19\%) exhibited morphologic features closer to T-CLL than to PLL and were termed the "small-cell variant." 7 However, there was no significant difference in clinical and laboratory features, response to chemotherapy, or survival between these 2 subgroups. Another series of 78 patients with T-PLL was reported by a French group in $1998 .^{2}$ The authors pointed out an initially indolent phase of disease, ranging from 6-103 months, which was present in about a third of the patients. With only 2 patients having CLLlike lymphoid morphology in the initially indolent subgroup and 2 patients in the initially aggressive subgroup, generalizations about the behavior of the CLL-like cases could not be made. A series of 25 patients with a mature T-cell phenotype and small, mature-appearing lymphocytes, termed "true T-cell CLL," was gathered from 3 major medical institutions over a 15-year period and published in $1995 .{ }^{8}$ Based largely on morphologic features and a lack of striking splenomegaly at diagnosis, an argument was advanced by the authors that T-CLL should continue

\footnotetext{
References

1. National Comprehensive Cancer Network. NCCN Guidelines Version 2.2014. Non-Hodgkin lymphoma. MS-275.. http:// www.nccn.org/professionals/physician_gls/pdf/nhl.pdf. Accessed September 2015.

2. Garand R, Goasguen J, Brizard A, et al. Indolent course as a relatively frequent presentation in T-prolymphocytic leukaemia. Groupe Français d'Hématologie Cellulaire. Br J Haematol. 1998;103:488-494.

3. Soma L, Cornfield DB, Prager D, Nowell P, Bagg A. Unusually indolent T-cell prolymphocytic leukemia associated with a complex karyotype: is this T-cell chronic lymphocytic leukemia? Am J Hematol. 2002;71:224-226.

4. Harris NL, Jaffe ES, Stein H, et al. A revised European-American classification of lymphoid neoplasms: a proposal from the
}

to be a separate designation in the classification of mature T-cell neoplasms. The authors did concede that their cases likely corresponded to the small-cell variant of T-PLL in the British series.

Our case does not fit neatly into any category of T-cell disorders in the present WHO classification of lymphomas. From a morphologic and behavioral standpoint, it seems nearly identical to Rai Stage 0 (Binet A) B-cell CLL, which may be present for decades before the need for therapy. ${ }^{1}$ The closest designation for our case in the 2008 WHO classification of lymphomas is the small-cell variant of T-PLL, an entity that does not differ significantly from the more common prolymphocytic morphologic form with respect to clinical presentation, poor response to therapy, and short (median, <1 year) survival according to the authors of the British series noted above. ${ }^{7}$ The longest survival in the French series of T-PLL was about 9 years, half the survival duration of our patient. ${ }^{2}$

In the original 2002 report of the present case, the authors suggested reviving the T-CLL designation. At this time, if T-CLL could be redefined to include cases with small, mature-appearing lymphoid morphology, a mature T-cell phenotype, and indolent rather than aggressive behavior, the classification would accommodate rare cases such as ours, which are presently termed T-PLL for lack of a more precise designation. Patients labelled as having T-PLL risk exposure to highly aggressive chemotherapy/immunotherapy regimens that may be inappropriate for indolent disease. There are presently no known phenotypic, cytogenetic, or molecular markers that can prospectively separate these rare indolent T-cell neoplasms from their more aggressive counterparts, a watch-and-wait approach to patients with T-PLL who do not exhibit aggressive disease, as exemplified by the current case, would appear to be prudent.

International Lymphoma Study Group. Blood. 1994;84:1361-1392. 5. Swerdlow SH, Campo E, Harris NL, et al. World Health

Organization Classification of tumours of haematopoietic and lymphoid tissues. Lyon, France: IARC Press; 2008.

6. Dungarwalla M, Matutes E, Dearden CE. Prolymphocytic leukaemia of B- and T-cell subtype: a state-of-the-art paper. Eur J Haematol. 2008;80:469-476.

7. Matutes E, Brito-Babapulle V, Swansbury J, et al. Clinical and laboratory features of 78 cases of T-prolymphocytic leukemia. Blood. 1991;78:3269-3274.

8. Hoyer JD, Ross CW, Li CY, et al. True T-cell chronic lymphocytic leukemia: a morphologic and immunophenotypic study of 25 cases. Blood. 1995;86:1163-1169. 